\title{
A cross-sectional study of the individual, social, and built environmental correlates of pedometer- based physical activity among elementary school children
}

\author{
Gavin R McCormack ${ }^{1 *}$, Billie Giles-Corti ${ }^{2}$, Anna Timperio ${ }^{3}$, Georgina Wood ${ }^{2}$ and Karen Villanueva ${ }^{2}$
}

\begin{abstract}
Background: Children who participate in regular physical activity obtain health benefits. Preliminary pedometerbased cut-points representing sufficient levels of physical activity among youth have been established; however limited evidence regarding correlates of achieving these cut-points exists. The purpose of this study was to identify correlates of pedometer-based cut-points among elementary school-aged children.

Method: A cross-section of children in grades 5-7 (10-12 years of age) were randomly selected from the most $(n=13)$ and least $(n=12)$ 'walkable' public elementary schools (Perth, Western Australia), stratified by socioeconomic status. Children $(n=1480$; response rate $=56.6 \%)$ and parents $(n=1332$; response rate $=88.8 \%)$ completed a survey, and steps were collected from children using pedometers. Pedometer data were categorized to reflect the sex-specific pedometer-based cut-points of $\geq 15000$ steps/day for boys and $\geq 12000$ steps/day for girls. Associations between socio-demographic characteristics, sedentary and active leisure-time behavior, independent mobility, active transportation and built environmental variables - collected from the child and parent surveys - and meeting pedometer-based cut-points were estimated (odds ratios: OR) using generalized estimating equations.
\end{abstract}

Results: Overall 927 children participated in all components of the study and provided complete data. On average, children took $11407 \pm 3136$ steps/day (boys: $12270 \pm 3350$ vs. girls: $10681 \pm 2745$ steps/day; p < 0.001) and 25.9\% (boys: 19.1 vs. girls: $31.6 \% ; \mathrm{p}<0.001$ ) achieved the pedometer-based cut-points.

After adjusting for all other variables and school clustering, meeting the pedometer-based cut-points was negatively associated $(p<0.05)$ with being male $(O R=0.42)$, parent self-reported number of different destinations in the neighborhood (OR 0.93), and a friend's (OR 0.62) or relative's (OR 0.44, boys only) house being at least a 10-minute walk from home. Achieving the pedometer-based cut-points was positively associated with participating in screen-time $<2$ hours/day (OR 1.88), not being driven to school (OR 1.48), attending a school located in a high SES neighborhood (OR 1.33), the average number of steps among children within the respondent's grade (for each 500 step/day increase: OR 1.29), and living further than a 10-minute walk from a relative's house (OR 1.69, girls only).

Conclusions: Comprehensive multi-level interventions that reduce screen-time, encourage active travel to/from school and foster a physically active classroom culture might encourage more physical activity among children.

Keywords: walkability youth, obesity, socioeconomic status, environment

\footnotetext{
* Correspondence: gmccorma@ucalgary.ca

'Population Health Intervention Research Centre, Department of Community

Health Sciences, University of Calgary, Alberta, Canada

Full list of author information is available at the end of the article
} 


\section{Background}

For children and adolescents, participation in regular physical activity provides physical and mental health benefits [1]. Specifically, regular physical activity can protect against weight gain and adiposity among children $[2,3]$, preventing the risk of adverse health outcomes including diabetes, hypertension, hyperlipidemia, depression, asthma, adverse orthopedic conditions and fatty-liver disease [4-6]. Several reviews have identified consistent correlates of physical activity among youth [7-10]. These correlates are multilevel - suggesting that a socio-ecological framework may best represent the determinants of physical activity among youth - and include biological (i.e., sex, ethnicity, age, parent weight status), psychological (i.e., self-efficacy, preferences, intention, attitude), behavioral (i.e., past activity, physical education, school sports), socio-cultural (i.e., parent activity and support, parent education, active siblings, friend support) and built environmental factors (i.e., access to facilities/programs, opportunities for exercise) $[7,8]$. Recent evidence shows there are associations between self-reported and objectively-assessed built environmental attributes and physical activity among children, including access to public recreational facilities (i.e., playgrounds/parks), transport infrastructure (i.e., sidewalks), and neighborhood safety and disorder (i.e., perceived safety and crime) $[11,12]$.

To date, much of the research examining correlates of physical activity among youth have relied on self-report methods for capturing physical activity behavior, including parent proxy. However, self-reported physical activity is prone to memory and recall bias $[13,14]$ and children's cognitive development may impair their ability to provide accurate recall [15]. Because they are relatively inexpensive and easy to use from both a study participant and research perspective, pedometers are increasingly being used to measure physical activity and as a motivational device in interventions designed to promote physical activity participation among children and adolescents $[16,17]$. Recently, various pedometerbased cut-points for children and adolescents have been suggested-primarily focusing on classification of those at risk of overweight and obesity [18-22]. These cutpoints (i.e., 13000-16000 steps/day) are within the normal range of daily pedometer-based physical activity undertaken by youth [23]. For instance, preliminary research examining pedometer-based cut-points among a sample of Australian, Swedish and United States youth (6-12 years of age) suggested that boys who participated in $\geq 15000$ steps/day and girls who participated in $\geq 12000$ steps/day were less likely to be classified as overweight or obese compared with children who took fewer steps [19]. Higher pedometer-based cut-points, including $\geq 16000$ steps/day for boys and $\geq 13000$ steps/ day for girls, have been reported where overweight was defined as a body fat percentage in the $85^{\text {th }}$ percentile [20] while lower pedometer-based cut-points among boys ( $\geq 13666$ steps/day) and girls ( $\geq 9983$ steps/day) 612 years of age have also been found [21].

Achieving any of the existing pedometer-based cutpoints does not guarantee that a child will be healthy weight. Nevertheless, boys achieving $\geq 15000$ steps/day and girls achieving $\geq 12000$ steps/day for instance, are more likely to participate in at least 60-minutes of moderate-intensity physical activity per day [24] - noteworthy given evidence showing a negative association between physical activity and body composition $[2,3,25]$. Moreover, the pedometer-based cut-points of $\geq 15000$ steps/day for boys and $\geq 12000$ steps/day for girls have been used as a benchmark for assessing physical activity levels among youth in several countries including Australia [26,27] and Canada [28] - the results of which indicate that over half of all children and adolescents are not achieving these levels. Yet, little is known about factors that may encourage or inhibit children achieving this level of pedometer-based physical activity.

Identifying factors that increase or decrease the likelihood of participating in or above these suggested pedometer-based cut-points may lead to more effective interventions designed to promote physical activity among children and adolescents. The limited evidence regarding correlates of achieving pedometer-based cutpoints suggests that sex, day of the week, age, participation in outside play, attending sports clubs, weather conditions, SES, participation in active transport and ethnicity may be important [29-33]. Nevertheless, other characteristics associated with meeting these recommendations may also exist, for instance, objectively or selfreported measures of the built and social environments.

Therefore the aim of this exploratory study is to extend the evidence-base by examining associations between socio-demographic characteristics, sedentary and active leisure-time behavior, independent mobility, active transportation and the built environment, and achievement of sex-specific pedometer-based cut-points [19], among Australian elementary school-aged children. Given the differential association between sex and achievement of the pedometer-based cut-points, the moderating effect of sex on the association between all other correlates and meeting this suggested benchmark will also be examined.

\section{Method}

This current study forms part of the TRavel, Environment, and Kids project (TREK). The overall aim of this project was to examine the extent to which the urban 
design of neighborhoods supports or discourages active transportation among children in grades 5-7 attending public elementary schools in Perth, Western Australia. The research methodology for TREK is presented elsewhere [34], and is briefly described here. Parent and child written informed consent was obtained for all participants. The University of Western Australia Human Research Ethics Committee granted ethics approval for this study.

\section{Sampling and recruitment}

Public elementary schools $(\mathrm{n}=238)$ across metropolitan Perth were assigned a school-specific walkability index and socioeconomic status (SES) score[34]. The walkability index reflected the street connectivity and traffic exposure within a $2 \mathrm{~km}$ walkable service area (defined using the street and informal pedestrian network). Connectivity of the walkable service area was measured by a Pedshed - a ratio of the pedestrian network area to the maximum possible area within a two kilometer Euclidian radius around the school. Vehicular traffic exposure comprised the sum of kilometers of each road type (including primary distributors ( $\geq 15000$ vehicles/day), district distributors ( $\geq 6000$ vehicles/day), and local distributors $(<6000$ vehicles/day) divided by kilometers of access roads ( $<3000$ vehicles/day)) within the walkable catchment area. School SES was based on the WA Department for Education and Training's 2001 Socioeconomic Index. Schools within each SES tertile (low, medium, and high) were ranked according to their walkbility score and the four top and bottom ranking schools within each tertile were invited to participate. Schools set in semi-rural locations and schools classified as 'high walkable' but located on a primary distributor road were excluded. Declining schools were replaced with the next ranked school. One additional high walkable/low SES school was recruited, due to the smaller number of children participating in this category. Overall, 69.4\% of schools approached agreed to participate $(n=25)$. One class from each grade 5-7 in each school was randomly selected until a minimum of 30 children per grade was recruited. Overall, 1480 children $(56.6 \%$ of all invited students) completed surveys, of those 1291 participated in the pedometer data collection and 1314 of their consenting parents also completed a self-administered survey.

\section{Data collection}

Data were collected July-December 2007 using a child questionnaire, parent questionnaire and pedometers in addition to other measures not reported here. Items were assessed for test-retest reliability between March and May 2007 and where available these estimates have been presented here as kappa $(\kappa)$ or intraclass correlation (ICC) coefficients.

\section{Pedometer-assessed physical activity}

Accusplit (AH120 M8) pedometers were used to record children's step counts. A similar model of pedometer was recently used to assess recommendations regarding moderate-intensity walking cadence [35]. The pedometers were worn level with the hip bone in line with the midpoint of the right knee. Children were asked to wear the pedometers at all times, except during water activities and while sleeping, for seven consecutive days including weekdays and weekend days. Children recorded whether they had worn the pedometer according to protocol each morning at school under the instruction of the classroom teacher. The in-built memory function negated the need for children to press reset each day and manually record step counts. After seven days, the pedometers were collected and the number of steps double punched by a data entry clerk to minimize data entry error.

\section{Socio-demographic variables}

Children reported whether they had friends in the neighborhood (i.e., many vs. few friends) while parents reported whether their household had a dog (i.e., owner vs. non-owner). The child's sex and grade were also collected in the children's questionnaire, and highest education for any parent (i.e., at least one parent in household with: high school or less, completed diploma/ college/technical school, or university education), marital status (i.e., married/defacto vs. other), the number of dependents $<18$ years at home (i.e., 1 child vs. $\geq 2$ children), and home ownership (i.e., renting vs. owned/purchasing) were assessed in the parent questionnaire.

\section{Sedentary and active leisure-time behavior}

Parents reported the average amount of time per day their child spent using a computer or internet for pleasure, watching television/videos, and playing passive or active electronic games. Minutes of each activity were summed and dichotomized to reflect current screenbased activity recommendations for Australian youth (i. e., $\leq 2$ vs. $>2$ hours/day)[36]. Children reported whether they had participated in the following leisure activities: playing in a park, playground, or playing field; playing team sport; attending a club or youth group; going for a walk in the neighborhood; playing in the street; playing in the yard; and taking the dog for a walk in the last week. An active leisure index was estimated representing the count of the different activities participated in by the child. To examine the possible effect of other students' physical activity levels on an individual child's physical activity behavior (i.e., peer influence), average pedometer steps within each grade within each school was also estimated. 


\section{Independent mobility and transportation to school}

Parents were asked whether or not their child was allowed to play in the street $(\kappa=0.56)$, play at the closest park, playground, or playing field $(\kappa=0.55)$, or walk in their neighborhood $(\kappa=0.59)$ without an adult. A dichotomous variable was derived from these items i.e., not allowed vs. allowed to play or walk in the neighborhood unsupervised. Parents also reported whether their child travelled to and from school by motor vehicle during a usual week $(\kappa=0.72)$.

\section{Self-reported neighborhood environment}

On a five-point Likert scale (i.e., strongly agree to strongly disagree) parents reported whether: they had to drive to get to a park with appealing equipment for their child; they often saw adults walking in their neighborhood; they often saw children walking in their neighborhood; their neighborhood was friendly; there was a lot of traffic in their neighborhood; the neighborhood was a nice place to walk around; there were safe crossings to reach a local shop; there were safe crossings to reach the closest local park; and drivers near the school often exceeded the speed limit (seven-day test-retest reliability $\mathrm{n}=89$ adults, $\mathrm{ICC}=0.42-0.66$ ). Parents also reported the time (i.e., $<5,5-10,11-15,16-20$, or $>20$ minutes) required for their child to walk from home to their nearest: primary school; shop; newsagent; library; transit station; park; bushland; sports field; beach, and; river. The number of different types of destinations perceived to be within a 10-minute from home was counted. The presence of a relative and friend's house within a 10-minute walk of home were examined separately. Reported time to walk to these destinations had acceptable reliability (ICC $=0.51-0.84)$.

\section{School neighborhood walkability and socioeconomic status}

The school-specific walkability index, as previously described [34], was dichotomized into high vs. low walkable. School area socioeconomic status (SES) was collapsed into tertiles (i.e., high, medium, and low SES).

\section{Data analysis}

The analytical sample included cases who participated in all components of the study and had complete survey and valid pedometer data $(\mathrm{n}=927)$. Pedometer-based physical activity was considered valid if counts were between 1000 and 30000 steps per day [37]-step counts outside this range were recoded as missing (5.9-32.0\% for any given day). Average daily steps was calculated for participants recording steps for at least four days [38]. Moderate inter-day reliability was found for pedometer data measured for four or more days (ICC = $0.65)$. Average daily steps were categorized into sex-specific pedometer-based cut-points $(<15000$ vs. $\geq 15000$ steps/day for boys and $<12000$ vs. $\geq 12000$ steps/day for girls) [19]. These cut-points were chosen in part because of their use as a benchmark in population-based physical activity surveys in Western Australia [26] and elsewhere [28] and because the cut-points were established using an international sample which included Australian youth [19].

Descriptive statistics were computed for all characteristics. Moreover, characteristics of the analytical sample $(\mathrm{n}=$ 927) and excluded ( $\mathrm{n}=553$; i.e., parent not participating in survey, child not participating in the pedometer data collection, or child not providing valid pedometer data) respondents were compared using Pearson's Chi-square and independent $\mathrm{t}$-tests. To reduce the number of parent neighborhood environment perception variables (i.e., drive to get to a park with appealing equipment for their child; often seeing adults walk in their neighborhood; often seeing children walk in their neighborhood; neighborhood is friendly; a lot of traffic in their neighborhood; neighborhood a nice place to walk around; safe crossings to reach a local shop; safe crossings to reach the closest local park, and; drivers near the school often exceeded the speed limit) a principal component analysis was performed. Items belonging to the same construct were identified (i.e., varimax rotated loadings $>0.40$ and on a single factor) and summed. Two factors were identified, including: 1$)$ neighborhood friendliness $(\mathrm{n}=4$ items; explained variance $=$ $30.9 \%$; factor loadings $=0.70-0.76$; Cronbach's alpha $=$ $0.74)$; and 2) traffic barriers ( $\mathrm{n}=4$ items; explained variance $=19.3 \%$; factor loadings $=0.58-0.78$; Cronbach's alpha $=0.66$ ). A single item-drive to get to a park with appealing equipment for their child-did not load on either factor and was examined separately in the analysis.

To account for school-level clustering, generalized estimating equations (GEE) were used (i.e., using an exchangeable correlation matrix and robust standard errors estimated) to estimate odds ratios (OR) and 95 percent confidence intervals $(95 \% \mathrm{CI})$. Using GEE, achievement of the pedometer-based cut-points was regressed onto the built environment, social environment, sedentary behavior, active leisure-time behavior, independent mobility, travel to school, and peer pedometer-based physical activity. To assess the moderating effect of sex, interaction terms between sex and the other correlates were estimated and a backward stepwise removal of non-significant interaction terms $(\mathrm{p}>0.05)$ undertaken to derive the final model. All main effects were retained in the model regardless of statistical significance. Based on bivariate correlations $(r<0.35)$ and variance inflation factors (VIFs $<1.4$ ) among the correlates, the level of multicollinearity within the regression model was not an issue. Statistical analysis was undertaken using Predictive Analytic Software 17 (SPSS, Inc).

\section{Results}

Compared with the excluded study participants, the analytical sample included a significantly higher proportion 
of girls, those spending $<2$ hours on screen-based activity/day, those allowed to play in the neighborhood without adult supervision, and those attending a school in a higher SES neighborhood (Table 1). Moreover, the analytical sample took more daily pedometer steps (11407 \pm 3136 vs. $10294 \pm 3891$ steps/day, $\mathrm{p}<0.001)$, were from grades that took a higher number of pedometer steps/ day $(11172 \pm 1231$ vs. $10846 \pm 1398, \mathrm{p}<0.001)$, participated in more leisure physical activities $(3.63 \pm 1.58$ vs. $3.45 \pm 1.66, \mathrm{p}=0.013)$, and had parents who reported a higher mix of destinations within a 10-minute walk of home $(5.45 \pm 2.48$ vs. $3.65 \pm 3.24, \mathrm{p}<0.001)$ compared

Table 1 Comparison of child and parent-reported questionnaire data and pedometer data among the analytical sample and cases excluded in the current study

\begin{tabular}{|c|c|c|c|c|}
\hline & & $\begin{array}{l}\text { Excluded cases } \\
\quad(n=553)\end{array}$ & $\begin{array}{c}\text { Analytical sample } \\
(\mathrm{n}=927)\end{array}$ & \\
\hline Variables & & $\%$ & $\%$ & $p$ \\
\hline \multirow[t]{2}{*}{ Home ownership* } & Renting & 28.8 & 23.9 & 0.084 \\
\hline & Owned/purchasing & 71.2 & 76.1 & \\
\hline \multirow[t]{2}{*}{ Marital status* } & Married/defacto & 80.5 & 78.2 & 0.370 \\
\hline & Other & 19.5 & 21.8 & \\
\hline \multirow[t]{2}{*}{ Dependents at home $<18 \mathrm{yrs}^{*}$} & One child & 10.7 & 12.6 & 0.370 \\
\hline & $\geq 2$ children & 89.3 & 87.4 & \\
\hline \multirow[t]{2}{*}{ Sex } & Male & 53.7 & 45.7 & 0.003 \\
\hline & Female & 46.3 & 54.3 & \\
\hline \multirow[t]{3}{*}{ Grade of student } & Grade 5 & 25.1 & 27.3 & 0.211 \\
\hline & Grade 6 & 34.4 & 36.8 & \\
\hline & Grade 7 & 40.5 & 35.9 & \\
\hline \multirow[t]{3}{*}{ Highest education completed by any parent* } & $\leq$ High school & 25.5 & 23.4 & 0.266 \\
\hline & Diploma/trade/other & 53.2 & 51.1 & \\
\hline & zBachelor degree & 21.3 & 25.5 & \\
\hline \multirow[t]{2}{*}{ Child usually driven to and from school } & No & 40.1 & 35.8 & 0.147 \\
\hline & Yes & 59.9 & 64.2 & \\
\hline \multirow[t]{2}{*}{ Screen-based activity time* } & $<2$ hours/day & 18.1 & 27.5 & $<0.001$ \\
\hline & $\geq 2$ hours/day & 81.9 & 72.5 & \\
\hline \multirow[t]{2}{*}{ Dog ownership * } & Owner & 55.2 & 57.1 & 0.547 \\
\hline & Non-owner & 44.8 & 42.9 & \\
\hline \multirow[t]{2}{*}{ Child has friends in the neighborhood } & Many friends & 69.5 & 68.7 & 0.762 \\
\hline & Few friends & 30.5 & 31.3 & \\
\hline \multirow[t]{2}{*}{ Distance to nearest friends house from home* } & $>10$ minute walk & 35.0 & 38.6 & 0.226 \\
\hline & $\leq 10$ minute walk & 65.0 & 61.4 & \\
\hline \multirow[t]{2}{*}{ Distance to nearest relatives house from home* } & $>10$ minute walk & 83.8 & 83.2 & 0.773 \\
\hline & $\leq 10$ minute walk & 16.2 & 16.8 & \\
\hline \multirow[t]{2}{*}{ Have to drive child to park with suitable equipment } & Yes & 39.9 & 35.9 & 0.179 \\
\hline & No & 60.1 & 64.1 & \\
\hline \multirow{2}{*}{$\begin{array}{l}\text { Play in any street, park, or go for walk without an } \\
\text { adult* }\end{array}$} & Not allowed & 20.2 & 14.0 & 0.006 \\
\hline & Allowed & 79.8 & 86.0 & \\
\hline \multirow[t]{2}{*}{ School neighborhood walkability score } & High & 47.7 & 45.6 & 0.431 \\
\hline & Low & 52.3 & 54.4 & \\
\hline \multirow[t]{3}{*}{ School neighborhood socioeconomic status } & High & 32.4 & 41.2 & $<0.001$ \\
\hline & Medium & 33.1 & 35.8 & \\
\hline & Low & 34.5 & 23.0 & \\
\hline \multirow[t]{2}{*}{ Pedometer-based physical activity } & $\begin{array}{l}\text { Not achieving suggested } \\
\text { steps }\end{array}$ & 79.5 & 74.1 & 0.058 \\
\hline & Achieving suggested steps & 20.5 & 25.9 & \\
\hline
\end{tabular}

* Indicates data collected from parent questionnaire; pedometer-based cut-points: $\geq 12000$ steps/day for girls and $\geq 15000$ steps/day for boys $\mathrm{N}=1480$ participated in child survey; $\mathrm{N}=1314$ participated in parent survey; $\mathrm{N}=1291$ children participated in pedometer data collection. Note: Excluded cases were those not participating in all components of the study as well as those not providing complete or valid data. 
with excluded participants (not shown in the Table). Parent perceived neighborhood traffic and friendliness did not significantly differ between the analytical and excluded samples (traffic: $2.90 \pm 0.78$ vs. $2.85 \pm 0.77$ and friendliness: $3.95 \pm 0.61$ vs. $3.90 \pm 0.63$, respectively). Excluding outliers, the minimum and maximum average daily steps for the analytical sample were 3164 and 22878 steps/day, respectively. Although boys on average took more daily steps than girls (boys: $12270 \pm 3350$ vs. girls: $10681 \pm 2745$ steps/day; $\mathrm{p}<0.001$ ), fewer boys achieved the pedometer-based cut-points (boys: 19.1 vs. girls: $31.6 \%$; $\mathrm{p}<0.001$ ).

\section{Correlates of pedometer-based cut-points}

The estimated exchangeable working correlation matrix suggested clustering of achievement of pedometer determined physical activity recommendations among students within the same school $(r=0.018$; number of respondents per grade: minimum $=7$; maximum $=65$ ) . Seven variables were associated $(\mathrm{p}<0.05)$ with achieving the suggested pedometer-based cut-points (female, $<2$ hours/day screen-based activity, not being driven to school, the average pedometer steps among other students within the child's school grade, the mix of neighborhood destinations perceived to be within 10 minutes from home, proximity of a friend's house, and higher school neighborhood SES) (Table 2). After adjustment, compared with girls, boys had lower odds of achieving the pedometer-based cut-points (OR 0.42). Participants had higher odds of achieving the pedometer-based cutpoints if they participated in less than two hours of screen-based activity/day (OR 1.88) or if they were not usually driven to and from school (OR 1.48). Moreover, the average number of steps among children within the respondent's grade was positively associated with achieving the pedometer-based cut-points. For each 500 steps/ day taken by students belonging to a child's grade at the same school, the odds of achieving the cut-points increased by $29 \%$ (OR 1.29) (Table 2). Noteworthy, was that the odds of achieving the pedometer-based cutpoints decreased as the parent perceived more destinations to be present within a 10-minute walk from home (OR 0.93). Moreover, children who resided more than 10-minutes from their friend's house had lower odds of achieving the pedometer-based cut-points (OR 0.62), while attending a school located in a high SES neighborhood was positively associated with achieving the cutpoints (OR 1.33). Notably, the overall walkability of the school walkable service area was not significantly associated with achieving the suggested pedometer-based cut-points.

The child's sex was found to moderate associations between distance to the closest relative's home and the average daily steps among all students within the respondent's grade at their school and achievement of the pedometer-based cut-points (Table 2). Sex-stratified models revealed that boys had lower odds (OR 0.44), while girls had higher odds (OR 1.69), of achieving the pedometer-based cut-points if they resided further than a 10-minute walk from a relative's home. Moreover, the association between the average pedometer-based physical activity among students within the respondent's grade and achievement of the pedometer-based cutpoints was slightly higher for boys (OR 1.43) than for girls (OR 1.23) (Table 3).

\section{Discussion}

This study provides new evidence adding to the existing literature on correlates of pedometer-based physical activity in children. We found that the achievement of pedometer-based cut-points [19] was associated with the child's own behavior (i.e., active transport to school, screen-based activity), the physical activity levels of their peers, and the characteristics of their neighborhood (i.e., school neighborhood-SES, proximity of a friend's house, the presence of local destinations).

Girls were more likely to achieve the suggested pedometer-based cut-points. Consistent with previous evidence [27-29,33] boys accumulated more daily steps than girls, yet they were less likely than girls to achieve this level of pedometer-based physical activity. Duncan et al. [39] found a similar pattern among 8-11 year old boys and girls. This discordance likely reflects the higher pedometer-based cut-point for boys ( $\geq 15000$ steps/day) compared with girls ( $\geq 12000$ steps). Only nineteen percent of boys and $32 \%$ of girls achieved the pedometerbased cut-point - somewhat lower than the prevalence for elementary school-aged children (grade 3, 5, and 7) found in a recent Western Australian survey (boys = $31.7 \%$ and girls $=43.9 \%$ ) [26]. However, the studies differed in a number of ways, including the specific grades captured, the sample design, the season in which data were collected, as well as the method for collecting and recording pedometer data (i.e., steps recorded daily vs. steps recorded after seven-days from the in-built memory). Nevertheless, our results correspond more closely with recent Australian national data suggesting that $24 \%$ of boys and 33\% of girls ages 9-13 achieved these same pedometer-based cut-points [27]. We found no association between school grade and achievement of the pedometer-based cut-points, consistent with studies that have found no relationship between steps and age among children [22,39].

Previous research has shown positive associations between maternal education, family income and physical activity levels among adolescents however, the relationship between SES and physical activity among children is less clear [8]. We found no association between 
Table 2 Odds ratios (OR) and $95 \%$ confidence intervals $(95 \% \mathrm{Cl})$ for the associations between socio-demographic, built environmental, and behavioral correlates of achieving pedometer-based cut-points

\begin{tabular}{|c|c|c|c|c|c|c|}
\hline \multirow{2}{*}{ Variables } & & \multirow[b]{2}{*}{$\%$} & \multicolumn{2}{|c|}{ Main effects } & \multicolumn{2}{|c|}{ Main effects + interactions } \\
\hline & & & OR & $95 \% \mathrm{Cl}$ & OR & $95 \% \mathrm{Cl}$ \\
\hline \multicolumn{7}{|l|}{ Socio-demographic } \\
\hline \multirow[t]{2}{*}{ Home ownership* } & Renting & 25.7 & 1.11 & $0.80,1.54$ & 1.11 & $0.80,1.56$ \\
\hline & Owned/purchasing & 26.0 & Ref. & & Ref. & \\
\hline \multirow[t]{2}{*}{ Marital status* } & Married/defacto & 25.5 & 1.00 & $0.59,1.69$ & 0.98 & $0.58,1.66$ \\
\hline & Other & 27.2 & Ref. & & Ref. & \\
\hline \multirow[t]{2}{*}{ Dependents at home $<18$ yrs* } & One child & 29.9 & 1.39 & $0.87,2.21$ & 1.42 & $0.89,2.26$ \\
\hline & $\geq 2$ children & 25.3 & Ref. & & Ref. & \\
\hline \multirow[t]{2}{*}{ Sex } & Male & 19.1 & 0.42 & $0.31,0.58^{\Phi}$ & 0.03 & $0.01,0.79^{\Phi}$ \\
\hline & Female & 31.6 & Ref. & & Ref. & \\
\hline \multirow[t]{3}{*}{ Grade of student } & Grade 5 & 28.1 & 1.12 & $0.81,1.54$ & 1.12 & $0.81,1.55$ \\
\hline & Grade 6 & 26.1 & 0.86 & $0.63,1.18$ & 0.90 & $0.65,1.25$ \\
\hline & Grade 7 & 24.0 & Ref. & & Ref. & \\
\hline \multirow{3}{*}{$\begin{array}{l}\text { Highest education completed by } \\
\text { any parent* }\end{array}$} & $\leq$ High school & 24.0 & 1.25 & $0.73,2.13$ & 1.28 & $0.77,2.13$ \\
\hline & Diploma/trade/other & 26.2 & 1.36 & $0.94,1.97$ & 1.38 & $0.95,2.03$ \\
\hline & $\geq$ Bachelor degree & 27.1 & Ref. & & Ref. & \\
\hline \multirow[t]{2}{*}{ Dog ownership* } & Owner & 26.1 & 1.08 & $0.75,1.55$ & 1.07 & $0.74,1.53$ \\
\hline & Non-owner & 25.6 & Ref. & & Ref. & \\
\hline \multirow{2}{*}{$\begin{array}{l}\text { Child has friends in the } \\
\text { neighborhood }\end{array}$} & Many friends & 27.8 & 1.04 & $0.73,1.49$ & 1.02 & $0.70,1.47$ \\
\hline & Few friends & 21.7 & Ref. & & Ref. & \\
\hline \multicolumn{7}{|l|}{$\begin{array}{l}\text { Sedentary and active leisure-time } \\
\text { behavior }\end{array}$} \\
\hline \multirow[t]{2}{*}{ Screen-based activity time* } & $<2$ hours/day & 35.7 & 1.88 & $1.30,2.73^{\Phi}$ & 1.89 & $1.29,2.78^{\Phi}$ \\
\hline & $\geq 2$ hours/day & 22.2 & Ref. & & Ref. & \\
\hline $\begin{array}{l}\text { Leisure physical activity index [min. } \\
=0, \max .=7] \wedge\end{array}$ & & & 1.08 & $0.95,1.36$ & 1.08 & $0.95,1.22$ \\
\hline $\begin{array}{l}\text { Average steps within grade }(500 \\
\text { step increments) }[\min .=7779.31 \\
\max .=14588.97] \wedge\end{array}$ & & & 1.29 & $1.22,1.36^{\Phi}$ & 1.21 & $1.13,1.31^{\Phi}$ \\
\hline \multicolumn{7}{|l|}{$\begin{array}{l}\text { Independent mobility and } \\
\text { transportation to school }\end{array}$} \\
\hline \multirow{2}{*}{$\begin{array}{l}\text { Play in any street, park, or go for } \\
\text { walk without an adult** }\end{array}$} & No & 22.3 & 0.71 & $0.44,1.14$ & 0.73 & $0.45,1.18$ \\
\hline & Yes & 26.5 & Ref. & & Ref. & \\
\hline \multirow{2}{*}{$\begin{array}{l}\text { Child usually driven to and from } \\
\text { school }\end{array}$} & No & 21.0 & 1.48 & $1.08,2.04^{\Phi}$ & 1.44 & $1.04,2.00^{\Phi}$ \\
\hline & Yes & 33.0 & Ref. & & Ref. & \\
\hline \multicolumn{7}{|l|}{$\begin{array}{l}\text { Self-reported neighborhood } \\
\text { environment }\end{array}$} \\
\hline \multirow{2}{*}{$\begin{array}{l}\text { Have to drive child to park with } \\
\text { suitable equipment }\end{array}$} & Yes & 27.1 & 0.99 & $0.72,1.35$ & 0.98 & $0.72,1.33$ \\
\hline & No & 23.7 & Ref. & & & \\
\hline $\begin{array}{l}\text { Traffic in neighborhood }[\min .=1 \\
\max .=5]^{*}\end{array}$ & & & 0.96 & $0.80,1.15$ & 0.97 & $0.81,1.16$ \\
\hline $\begin{array}{l}\text { Friendliness of neighborhood [min. } \\
=1, \max .=5]^{*}\end{array}$ & & & 1.15 & $0.94,1.41$ & 1.18 & $0.94,1.47$ \\
\hline \multirow{2}{*}{$\begin{array}{l}\text { Distance to nearest friends house } \\
\text { from home* }\end{array}$} & $>10$ minute walk & 20.4 & 0.62 & $0.47,0.82^{\Phi}$ & 0.60 & $0.46,0.78^{\Phi}$ \\
\hline & $\leq 10$ minute walk & 29.3 & Ref. & & Ref. & \\
\hline \multirow{2}{*}{$\begin{array}{l}\text { Distance to nearest relatives house } \\
\text { from home* }\end{array}$} & $>10$ minute walk & 26.1 & 1.05 & $0.61,1.80$ & 1.66 & $0.97,2.85$ \\
\hline & $\leq 10$ minute walk & 25.0 & Ref. & & Ref. & \\
\hline
\end{tabular}


Table 2 Odds ratios (OR) and $95 \%$ confidence intervals $(95 \% \mathrm{Cl})$ for the associations between socio-demographic, built environmental, and behavioral correlates of achieving pedometer-based cut-points (Continued)

\begin{tabular}{|c|c|c|c|c|c|c|}
\hline $\begin{array}{l}\text { Count of different destinations } \\
\text { within a } 10 \text { minute walk of home } \\
{[\text { min. }=0 \text {, max. }=13]^{*}}\end{array}$ & & & 0.93 & $0.88,0.99^{\Phi}$ & 0.93 & $0.88,0.99^{\Phi}$ \\
\hline \multicolumn{7}{|l|}{$\begin{array}{l}\text { Neighborhood walkability and } \\
\text { socioeconomic status }\end{array}$} \\
\hline \multirow{2}{*}{$\begin{array}{l}\text { School neighborhood walkability } \\
\text { score }\end{array}$} & High & 28.6 & 1.01 & $0.75,1.35$ & 1.00 & $0.74,1.35$ \\
\hline & Low & 23.6 & Ref. & & Ref. & \\
\hline \multirow{3}{*}{$\begin{array}{l}\text { School neighborhood } \\
\text { socioeconomic status }\end{array}$} & High & 29.6 & 1.33 & $1.06,1.67^{\Phi}$ & 1.36 & $1.11,1.68^{\Phi}$ \\
\hline & Medium & 25.0 & 1.15 & $0.82,1.60$ & 1.15 & $0.82,1.60$ \\
\hline & Low & 20.7 & Ref. & & Ref. & \\
\hline \multicolumn{7}{|l|}{ Sex-specific interactions } \\
\hline \multicolumn{2}{|l|}{$\begin{array}{l}\text { Sex by dist. relatives house within } \\
\text { 10-min walk }\end{array}$} & & & & 0.27 & $0.15,0.47^{\Phi}$ \\
\hline \multicolumn{2}{|l|}{ Sex by average steps within grade } & & & & 1.17 & $1.02,1.34^{\Phi}$ \\
\hline
\end{tabular}

household SES-parent education and home ownershipand achievement of the pedometer-based cut-points. Children attending a school in a higher socioeconomic neighborhood were more likely to achieve the pedometer-based cut-points compared with those attending schools in low socioeconomic neighborhoods, even after accounting for household indicators of SES. Disadvantaged neighborhoods often offer fewer physical activity opportunities for children [40]. While our walkability index captured pedestrian connectivity and traffic exposure, it is possible that other environmental characteristics related to neighborhood SES exist (e.g., land-use mix, aesthetics, and safety).

Level of independent mobility, such as going to local shops, school, friends, or park unsupervised, may be associated with increased outdoor play, participation in structured sport and exercise, and walking and cycling to and from school $[41,42]$. As well as potentially decreasing physical activity opportunities, restricting independent mobility may negatively influence a child's cognitive and social development by reducing opportunities to socialize and explore and interact with their neighborhood environment [43,44]. Moreover, children with restricted mobility may spend more time participating in passive modes of transportation, particularly for travel to school [45]. Although children who were not allowed to play in their neighborhood unsupervised were less likely to achieve the pedometerbased cut-points, this was not statistically significant. Nevertheless, we did find that children who were usually driven to or from school were less likely to achieve the pedometer-based cut-point, suggesting that active transportation such as walking can contribute to overall levels of physical activity. Positive associations between levels of active transportation and physical activity, including pedometer-based physical activity among youth have been reported elsewhere $[46,47]$. Creating safe child-friendly neighborhoods, changing parent and child perceptions and attitudes towards motor vehicle use, encouraging parents to walk or be active in their neighborhoods with their children, and implementing sustainable walk to school programs could encourage

Table 3 Sex-stratified models showing odds ratios (OR)* and $95 \%$ confidence intervals (95\%Cl) of the association between average steps within the respondent's grade, distance to nearest relatives house from home, and achievement of pedometer-based cut-points

\begin{tabular}{|c|c|c|c|c|c|}
\hline \multirow[b]{3}{*}{ Variables } & & \multicolumn{4}{|c|}{ Achieving the pedometer-based cut-points } \\
\hline & & \multicolumn{2}{|c|}{ Boys $(n=424)$} & \multicolumn{2}{|c|}{ Girls ( $n=503)$} \\
\hline & & OR & $95 \% \mathrm{Cl}$ & OR & $95 \% \mathrm{Cl}$ \\
\hline Average steps within grade (in 500 step increments) & & 1.43 & $1.27,1.60^{\Phi}$ & 1.23 & $1.14,1.33^{\Phi}$ \\
\hline \multirow[t]{2}{*}{ Distance to nearest relatives house from home* } & $>10$ minute walk & 0.44 & $0.22,0.86^{\Phi}$ & 1.69 & $0.98,2.90$ \\
\hline & $\leq 10$ minute walk & Ref. & & & \\
\hline
\end{tabular}

*Adjusted for all built environmental, social environmental, socioeconomic, demographic, and behavioral correlates presented in Table 2 .

$\Phi_{p}<0.05$. Ref.: reference category. 
independent mobility and active transportation which may contribute to increasing levels of physical activity.

There has been a recent focus on the independent contribution of sedentary behavior on the health of both adults and children $[48,49]$. We found that children who spent more than two hours daily on screen-based activity took fewer steps and were less likely to achieve the pedometer-based cut-points. Our finding is important as only modest or null associations between time spent in screen-based activity and steps among children have been previously found [49-51]. Interventions that decrease screen-based activity could contribute to higher levels of physical activity participation among children.

School neighborhood walkability and parent perceptions of traffic barriers and safety or neighborhood friendliness were not associated with pedometer-based physical activity. We did find, however, a negative relationship between the count of different neighborhood destinations that parents perceive to be within a 10minute walk of home and achieving the pedometerbased cut-points. Some destinations captured in this study might be considered less important for encouraging children to be more physically active (e.g., libraries and news agencies). The presence of more local destinations might also result in the neighborhood being less supportive of physical activity among children of this age because of more destination-related motor vehicle traffic, limited area for play spaces and the presence of more strangers visiting local neighborhoods. An alternative explanation is that fewer steps are needed to travel to destinations that are closer to home. Nevertheless, Frank et al. [52], for example, found objectively-assessed mix of land uses to be positively associated with walking among adolescents but not children aged 5-11 years. Furthermore, others have noted negative associations between environmental characteristics usually supportive of physical activity among adults (i.e., street connectivity) and walking in children [53]. The lack of association between the perceived neighborhood environment and pedometer-based physical activity is not surprising as evidence of this relationship among pre-adolescent children is mixed [54].

Children whose friend's house was within a 10-minute walk were more likely to achieve pedometer-based cutpoints. Children with friends residing nearby might visit more frequently using active modes, may be involved in active play, and may have company to walk to and from school. Together this may result in more walking and physical activity. There is evidence for associations between friend support and physical activity among children [8]. In support, we also found a positive association between a child's pedometer-based physical activity and the physical activity level of their peers (within the same grade level at their school) - with the association slightly stronger for boys than girls. Peer physical activity levels might encourage physical activity behaviors among youth [55]. Our finding could reflect peer modeling or support, or unmeasured influences of the school (i.e., quantity and quality of physical education or school sports) or the neighborhood environment on physical activity. In addition, boys were less likely to achieve the pedometer-based cut-point if they resided further than a 10-minute walk of their nearest relative's home, whereas girls were more likely to achieve the cut-point. Speculatively, girls might be more willing to walk further than boys to visit relatives resulting in higher recorded pedometer-based physical activity. More research is needed to disentangle these associations.

Several limitations should be considered when interpreting the findings of this study. Differences between the analytical sample and the excluded participants were observed. In particular the analytical sample was more physically active, thus the results presented might underestimate the magnitude of the associations between the correlates and pedometer-based physical activity. Moreover, the moderate reliability of some variables may have attenuated the estimated associations between these correlates and the outcome. The generalizability of these results are limited to school-aged children in grades 5-7 attending elementary schools in high and low walkable neighborhoods determined based on pedestrian connectivity and traffic exposure. Other objectively-assessed environmental characteristics not measured here could be important for determining pedometer steps among children, although more research is needed to identify these characteristics. We examined the correlates of one set of recently suggested pedometer-based physical activity cut-points [19], thus the associations found here may not generalize to other existing pedometer-based cut-points for children and adolescents. Children and adolescents achieving the pedometer-based cut-points used in this study may be less likely to be classified as overweight and obese compared with those not achieving these cut-points [19] however, we did not examine the association between steps and weight status or any other health outcome. Future research should investigate the extent to which achievement of the various established pedometer-based physical activity cut-points result in positive health outcomes including, but not limited to, improved weight status among children and adolescents. Finally, associations found from this cross-sectional study cannot be considered causal.

\section{Conclusions}

For children, participation in regular physical activity can provide physical and mental health benefits and physical activity behaviors adopted during childhood 
often track into adulthood [56]. These results suggest that children, who participate in less screen-based activities at home, use active modes to travel to and from school and attend schools that foster a physically active classroom culture take more pedometer-based steps daily. Multi-level interventions, programs, and policies are required particularly targeting lower socioeconomic schools. More research examining correlates of pedometer-based physical activity separately for boys and girls is needed.

\section{Abbreviations}

(BMI): body mass index; (ICC): intraclass correlation; (OR): odds ratio; (SES): socioeconomic status; $(95 \% \mathrm{Cl})$ : 95 percent confidence interval;

\section{Acknowledgements}

This study was funded by the NHMRC (ID\#403933). GRM is supported by a Canadian Institutes of Health Research Bisby Postdoctoral Fellowship Award. BGC is supported by an Australian NHMRC Senior Research Fellowship (\#503712). AT is supported by a Vic Health Public Health Research Fellowship. GW is supported by scholarships provided by an Australian Postgraduate Award and a NHMRC Capacity Building Grant (\#458668).

\section{Author details}

'Population Health Intervention Research Centre, Department of Community Health Sciences, University of Calgary, Alberta, Canada. ${ }^{2}$ Centre for Built Environment, School of Population Health, University of Western Australia, Australia. ${ }^{3}$ Centre for Physical Activity and Nutrition Research, Deakin University, Melbourne, Victoria, Australia.

\section{Authors' contributions}

BGC, AT, and GRM conceived this study. GW and KW contributed to data collection. GRM conducted the analysis. All authors contributed to interpretation and writing of the manuscript. All authors read and approved the final manuscript

\section{Competing interests}

The authors declare that they have no competing interests.

Received: 23 September 2010 Accepted: 12 April 2011

Published: 12 April 2011

\section{References}

1. Biddle SJ, Gorely T, Stensel DJ: Health-enhancing physical activity and sedentary behaviour in children and adolescents. J Sports Sci 2004, 22:679-701.

2. Atlantis E, Barnes EH, Singh MA: Efficacy of exercise for treating overweight in children and adolescents: a systematic review. Int J Obes 2006, 30:1027-1040.

3. Janssen I, Katzmarzyk PT, Boyce WF, Vereecken C, Mulvihill C, Roberts C, Currie C, Pickett W: Comparison of overweight and obesity prevalence in school-aged youth from 34 countries and their relationships with physical activity and dietary patterns. Obes Rev 2005, 6:123-132.

4. Lobstein T, Jackson-Leach R: Estimated burden of pediatric obesity and co-morbidities in Europe. Part 2. Numbers of children with indicators of obesity-related disease. Int I Pediatr Obes 2006, 1:33-41.

5. Daniels SR, Arnett DK, Eckel RH, Gidding SS, Hayman LL, Kumanyika S, Robinson TN, Scott BJ, St Jeor S, Williams CL: Overweight in children and adolescents: pathophysiology, consequences, prevention, and treatment. Circulation 2005, 111:1999-2012.

6. Torrance B, McGuire KA, Lewanczuk R, McGavock J: Overweight, physical activity and high blood pressure in children: a review of the literature. Vasc Health Risk Manag 2007, 3:139-149.

7. Sallis JF, Prochaska JJ, Taylor WC: A review of correlates of physical activity of children and adolescents. Med Sci Sports Exerc 2000, 32:963-975.
8. Van Der Horst K, Paw MJ, Twisk JW, Van Mechelen W: A brief review on correlates of physical activity and sedentariness in youth. Med Sci Sports Exerc 2007, 39:1241-1250.

9. Gustafson SL, Rhodes RE: Parental correlates of physical activity in children and early adolescents. Sports Med (Auckland, N Z) 2006, 36:79-97.

10. van der Horst K, Oenema A, Ferreira I, Wendel-Vos W, Giskes K, van Lenthe F, Brug J: A systematic review of environmental correlates of obesity-related dietary behaviors in youth. Health Educ Res 2007, 22:203-226.

11. Davison KK, Lawson CT: Do attributes in the physical environment influence children's physical activity? A review of the literature. Int J Behav Nutr Phys Act 2006, 3:19.

12. Carver A, Timperio A, Crawford D: Playing it safe: the influence of neighbourhood safety on children's physical activity. A review. Health Place 2008, 14:217-227

13. Welk GJ, Corbin CB, Dale D: Measurement issues in the assessment of physical activity in children. Res Q Exerc Sport 2000, 71:S59-73.

14. Sallis JF, Saelens BE: Assessment of physical activity by self-report: status, limitations, and future directions. Res Q Exerc Sport 2000, 71:S1-14.

15. Baranowski T: Validity and reliability of self-report measures of physical activity: An information-processing perspective. Res Q Exerc Sport 1988, 59:314-327.

16. Butcher Z, Fairclough S, Stratton G, Richardson D: The effect of feedback and information on children's pedometer step counts at school. Pediatr Exerc Sci 2007, 19:29-38.

17. Lubans DR, Morgan PJ, Tudor-Locke C: A systematic review of studies using pedometers to promote physical activity among youth. Prev Med 2009, 48:307-315

18. Eisenmann JC, Laurson KR, Wickel EE, Gentile D, Walsh D: Utility of pedometer step recommendations for predicting overweight in children. Int J Obes 2007, 31:1179-1182.

19. Tudor-Locke C, Pangrazi RP, Corbin CB, Rutherford WJ, Vincent SD, Raustorp A, Tomson LM, Cuddihy TF: BMI-referenced standards for recommended pedometer-determined steps/day in children. Prev Med 2004, 38:857-864

20. Duncan JS, Schofield G, Duncan EK: Step count recommendations for children based on body fat. Prev Med 2007, 44:42-44.

21. Laurson KR, Eisenmann JC, Welk GJ, Wickel EE, Gentile D, Walsh D: Evaluation of youth pedometer-determined of youth pedometerdetermined physical activity guidelines using receiver operator characteristics curves. Prev Med 2008, 46:419-424.

22. Vincent SD, Pangrazi RP: An examination of the activity patterns of elementary school children. Pediatr Exerc Sci 2002, 14:432-441.

23. Tudor-Locke C, MCClain JJ, Hart TL, Sisson SB, Washington TL: Expected values for pedometer-determined physical activity in youth. Res $Q$ Exerc Sport 2009, 80:164-174.

24. Rowlands AV, Eston RG: Comparison of accelerometer and pedometer measures of physical activity in boys and girls, ages 8-10 years. Res $Q$ Exerc Sport 2005, 76:251-257.

25. Ekelund U, Sardinha LB, Anderssen SA, Harro M, Franks PW, Brage $S$, Cooper AR, Andersen LB, Riddoch C, Froberg K: Associations between objectively assessed physical activity and indicators of body fatness in 9- to 10-y-old European children: a population-based study from 4 distinct regions in Europe (the European Youth Heart Study). Am J Clin Nutr 2004, 80:584-590.

26. Martin K, Rosenberg M, Miller M, French S, McCormack G, Bull F, GilesCorti B, Pratt S: Child and Adolescent Physical Activity and Nutrition Survey 2008: Key Findings. Perth: Western Australian Government; 2009.

27. Commonwealth Scientific Industrial Research Organisation (CSIRO) Preventative Health National Research Flagship: 2007 Australian National Children's Nutrition and Physical Activity Survey: Main Findings. Adelaide: Prepared by Commonwealth Scientific Industrial Research Organisation (CSIRO) Preventative Health National Research Flagship, and the University of South Australia; 2008.

28. Craig C, Cameron C, Griffiths J, Tudor-Locke C: Descriptive epidemiology of youth pedometer-determined physical activity: CANPLAY. Med Sci Sports Exerc 2010, 42:1639-1643.

29. Le Masurier G, Beighle A, Corbin C, Darst P, Morgan C, Pangrazi R, Wilde B, Vincent S: Pedometer-determined physical activity levels of youth. J Phys Act Health 2005, 2:159-168. 
30. Duncan JS, Hopkins WG, Schofield G, Duncan EK: Effects of weather on pedometer-determined physical activity in children. Med Sci Sports Exerc 2008, 40:1432-1438.

31. Duncan EK, Scott Duncan J, Schofield G: Pedometer-determined physical activity and active transport in girls. Int J Behav Nutr Phys Act 2008, 5:2.

32. Hohepa M, Schofield G, Kolt GS, Scragg R, Garrett N: Pedometerdetermined physical activity levels of adolescents: differences by age, sex, time of week, and transportation mode to school. J Phys Act Health 2008, 5(Suppl 1):S140-152.

33. Loucaides C, Jago R: Correlates of pedometer-assessed physical activity in Cypriot elementary school children. J Phys Act Health 2006, 3:267-276.

34. Giles-Corti B, Wood G, Pikora T, Learnihan V, Bulsara M, Van Niel K, Timperio A, McCormack G, Villanueva K: School site and the potential to walk to school: The impact of street connectivity and traffic exposure in school neighborhoods. Health Place 17:545-530

35. Rowe D, Welk G, Heil D, Mahar M, Kemble C, Calabró M, Camenisch K Stride rate recommendations for moderate intensity walking. Med SCi Sports Exerc 2011, 43(2):312-8.

36. Commonwealth of Australia: Active Kids are Healthy Kids. Australia's Physical Activity Recommendations for 5-12 Year Olds. Canberra: Department of Health and Ageing, Commonwealth of Australia; 2004

37. Rowe DA, Mahar MI, Raedeke TD, Lore J: Measuring physical activity in children with pedometers: Reliability, reactivity, and replacement of missing data. Pediatr Exerc Sci 2004, 16:343-354.

38. Strycker LA, Duncan SC, Chaumeton NR, Duncan TE, Toobert DJ: Reliability of pedometer data in samples of youth and older women. Int I Behav Nutr Phys Act 2007, 4:4.

39. Duncan MJ, Al-Nakeeb Y, Woodfield L, Lyons M: Pedometer determined physical activity levels in primary school children from central England. Prev Med 2007, 44:416-420.

40. Crawford D, Timperio A, Giles-Corti B, Ball K, Hume C, Roberts R, Andrianopoulos N, Salmon J: Do features of public open spaces vary according to neighbourhood socio-economic status? Health Place 2008, 14:889-893.

41. Wen LM, Kite J, Merom D, Rissel C: Time spent playing outdoors after school and its relationship with independent mobility: a cross-sectional survey of children aged 10-12 years in Sydney, Australia. Int J Behav Nutr Phys Act 2009, 6:15.

42. Page AS, Cooper AR, Griew P, Jago R: Independent mobility, perceptions of the built environment and children's participation in play, active travel and structured exercise and sport: the PEACH Project. Int J Behav Nutr Phys Act 2010, 7:17.

43. Prezza M, Pilloni S, Morabito C, Sersante C, Alparone FR, Giuliani MV: The influence of psychosocial and environmental factors on children's independent mobility and relationship to peer frequentation. J Community App/ Soc 2001, 11:435-450

44. Risotto A, Tonucci F: Freedom of movement and environmental knowledge in elementary school children. J Environ Psychol 2002, 22:65-77.

45. Merom D, Tudor-Locke C, Bauman A, Rissel C: Active commuting to school among NSW primary school children: implications for public health. Health Place 2006, 12:678-687.

46. Faulkner GE, Buliung RN, Flora PK, Fusco C: Active school transport, physical activity levels and body weight of children and youth: a systematic review. Prev Med 2009, 48:3-8.

47. Lee $M$, Orenstein M, Richardson M: Systematic review of active commuting to school and children's physical activity and weight. J Phys Act Health 2008, 5:930-949.

48. Owen N, Healy G, Matthews C, Dunstan D: Too much sitting: the population health science of sedentary behavior. Exerc Sport Sci Rev 2010, 38:105-113.

49. Must A, Tybor DJ: Physical activity and sedentary behavior: a review of longitudinal studies of weight and adiposity in youth. Int J Obes 2005, 29(Suppl 2):S84-96.

50. Ziviani J, Macdonald D, Ward H, Jenkins D, Rodger S: Physical activity of young children: a two-year follow-up. Phys Occup Ther Pediatr 2008, 28:25-39.

51. Laurson KR, Eisenmann JC, Welk GJ, Wickel EE, Gentile DA, Walsh DA: Combined influence of physical activity and screen time recommendations on childhood overweight. J Pediatr 2008, 153:209-214.
52. Frank L, Kerr J, Chapman J, Sallis J: Urban form relationships with walk trip frequency and distance among youth. Am J Health Promot 2007, 21:305-311.

53. Timperio A, Ball K, Salmon J, Roberts R, Giles-Corti B, Simmons D, Baur LA, Crawford D: Personal, family, social, and environmental correlates of active commuting to school. Am J Prev Med 2006, 30:45-51.

54. Ferreira I, van der Horst K, Wendel-Vos W, Kremers S, van Lenthe FJ, Brug J: Environmental correlates of physical activity in youth - a review and update. Obes Rev 2007, 8:129-154.

55. Vilhjalmsson $\mathrm{R}$, Thorlindsson $\mathrm{T}$ : Factors related to physical activity: a study of adolescents. Soc Sci Med 1998, 47:665-675.

56. Telama R, Yang X, Viikari J, Valimaki I, Wanne O, Raitakari O: Physical activity from childhood to adulthood: A 21-year tracking study. Am J Prev Med 2005, 28:267-273.

doi:10.1186/1479-5868-8-30

Cite this article as: McCormack et al:: A cross-sectional study of the individual, social, and built environmental correlates of pedometerbased physical activity among elementary school children. International Journal of Behavioral Nutrition and Physical Activity 2011 8:30.

\section{Submit your next manuscript to BioMed Central and take full advantage of:}

- Convenient online submission

- Thorough peer review

- No space constraints or color figure charges

- Immediate publication on acceptance

- Inclusion in PubMed, CAS, Scopus and Google Scholar

- Research which is freely available for redistribution
C BioMed Central 